\title{
Iris Surface Deformation and Normalization
}

\author{
Somying Thainimit \\ Electrical Engineering Department \\ Faculty of Engineering \\ Kasetsart University \\ Bangkok, Thailand \\ fengsyt@ku.ac.th
}

\author{
Luís A. Alexandre \\ Department of Informatics \\ University of Beira Interior \\ IT-Instituto de Telecomunicações \\ Covilhã, Portugal \\ luis.alexandre@ubi.pt
}

\author{
Vasco M. N. de Almeida \\ Department of Physics \\ University of Beira Interior, Covilhã \\ Centre of Physics \\ University of Minho, Braga \\ Portugal \\ vasco@ubi.pt
}

\begin{abstract}
Human iris recognition systems are an attractive form of non-intrusive bio-identification with many potential applications. However, the accuracy of these systems is still limited due to challenges presented by iris surface deformation. This paper provides an introduction to the physiology of the iris, describes the problem of iris deformation, and presents a review of past and current software approaches to iris recognition. The focus is on methods related to the physiology of the iris.
\end{abstract}

Keywords-iris surface deformation; iris normalization; iris recognition;

\section{INTRODUCTION}

Deformations of the iris are primary changes that affect structures of the iris surface. Since iris recognition system identifies or verifies the identity of a person based on iris surface structures or patterns, iris surface deformation degrades performance of the recognition system significantly. Previous studies [1,2] reported that the performance of iris recognition systems degrades substantially when enrolled iris images and live scanned images of the same iris have large differences in the radius of the pupil. The percent of incorrectly reject valid individual (False non-match rate) is higher when pupil changes in size. Thus, this problem needs to be addressed to make a robust iris recognition system.

Size variations of the iris can caused from capturing iris images at different distances or angles and from pupil movements. For the former case, the iris surface deformation can be handled using conventional camera transformations. However, addressing a change of iris size caused by pupil movements such as pupil dilations, contractions and other physiological phenomena is an ongoing research. Current knowledge of physiological mechanisms that deform the iris surface is still limited and needs further investigation.

This paper described background and research related to physiology based iris deformation and normalization in order to facilitate the work and encourage more scientists into this field. Section 2 covers the background of the anatomy and physiology of the human iris and pupil. Relevant medical terminology is described in order to provide a background for computer scientists who are not familiar with the medical terms. Section 3 presents a review of existing iris deformation models used in the iris recognition literature. Section 4 provides an introductory review of existing iris normalization schemes. Section 5 contains our discussion and conclusion.

\section{HUMAN IRIS AND PUPIL}

\section{A. The Iris}

The human iris is a visible color ring bounded by the pupil (the dark opening) and white sclera, as depicted in Fig. 1. The iris is suspended in aqueous humor, behind the cornea but in front of the lens as shown in Fig. 2. The iris divides the space between the lens and the cornea into the anterior chamber (between the cornea and the iris) and posterior chambers (between the iris and the lens). The periphery of the iris is attached to its root at the iridocorneal angle of the anterior chamber where it merges with the tissue inside the eye: the ciliary body and trabecular meshwork. The free edge of the iris is known as the pupillary margin or pupillary ruff (the boundary of the pupil). The formation of the human iris begins during the third month of gestation; its distinguishable pattern is completed by the eight month of gestation, but pigmentation continues into the first year after birth [3]. The size of the iris varies from person to person with a range of 10.2 to $13.0 \mathrm{~mm}$ in diameter [4], an average size of $12 \mathrm{~mm}$ in diameter, and a circumference of $37 \mathrm{~mm}$ [5].

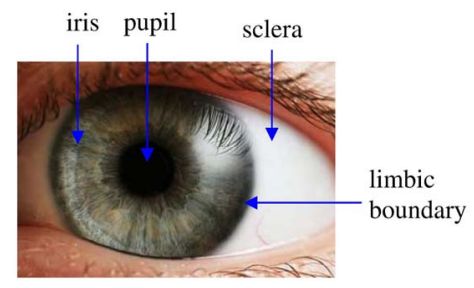

Fig.1. Anterior surface of the iris

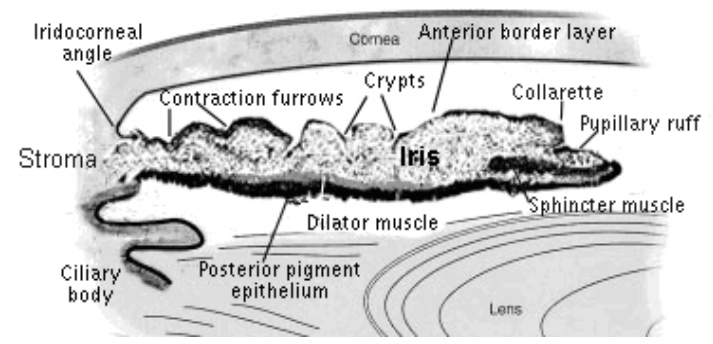

Fig.2. Anterior segment of the eye (Modified from [6]).

This work was supported by 'FCT-Fundação para a Ciência e Tecnologia' and 'FEDER' in the scope of the PTDC/EIA-EIA/103945/2008 research project 'NECOVID: Covert Negative Biometric Identification'. 
The microscopic anatomy of the iris consists of four layers (from front to back): the anterior border layer, stroma and the sphincter muscle fibers, dilator muscle fibers, and the posterior pigment epithelium, as shown in Fig. 2.

The anterior border layer is the visible part of the iris. It is lightly pigmented. Aggregates of heavily pigmented melanocytes (melanin producing cells) in this layer are called naevi or freckles. The stroma consists of loose connective tissue containing fibroblasts, melanocytes, and collagen fibers. These connected fibers create meshes in which the blood vessels and nerves are integrated. The pigment content (composed of melanin and lipochrome) within the stroma and the anterior layer plays important roles in the appearance of eye color $[6,7]$. A brown iris contains more pigment on its anterior surface and within its stroma than a blue iris.

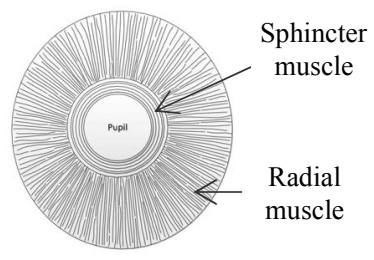

Fig.3. Sphincter and dilator pupillae muscles.

Lying within the stroma close to the pupil margin is the sphincter pupillae or circular muscle. The muscle consists of involuntary smooth muscle fibers circularly arranged near the pupil margin as illustrated in Fig. 3. The muscle band is about 0.75 to $1 \mathrm{~mm}$ in diameter and 0.1 to $1.7 \mathrm{~mm}$ in thickness [8]. The dilator pupillae muscles (radial muscles) form a flat sheet immediately beneath the stroma. The muscle is about $4 \mu \mathrm{m}$ in thickness. It extends in a radial direction from the border of the iris (iris root) to the border of the sphincter. These two groups of smooth muscles control the size of pupil, which regulates the amount of light entering the eye.

The posterior epithelium includes two layers of cells heavily pigmented. Most parts of the epithelial layer are invisible except for the pigment frill or pupillary ruff and crypts. The pupillary ruff is derived from a short forward extension of the posterior epithelium layer onto the anterior iris surface at the pupil margin. During slit lamp examination of the eye, the pupillary ruff appears as a dark ring nearby the pupil margin. The crypts are openings caused from discontinuity of the anterior layer over the posterior layer. These openings reveal dark posterior pigmented cells. The posterior epithelium layer also forms a series of radially arranged furrows seen near the pupil margin and circumferential contraction folds (often seen near the iris periphery).

The anterior surface of the iris is divided into two zones: the pupillary and the ciliary zone (see Fig. 4). These two zones are separated by the collarette, which is the thickest part of the iris (about $0.6 \mathrm{~mm}$ ). The pupillary zone is an inner region with its edge forming the boundary of the pupil. The rest of the iris that extends to its origin at the ciliary body (iris root) is the ciliary zone. The anterior surface of the iris contains several distinct features such as crypts, pigments spots, folds, and furrows.

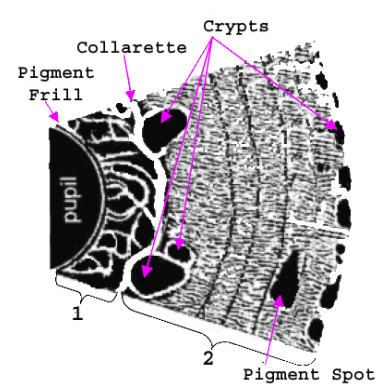

Fig.4. An anterior iris surface [3]:1= pupillary zone, $2=$ ciliary zone.

The posterior surface of the iris is smoother than the anterior one. The smooth surface is interrupted by relatively shallow furrows or folds such as the contraction folds of Schwalbe, the structural folds of Schwalbe, and the circular contraction folds [8]. A combination of both anterior and posterior surface features provides highly complex and unique iris patterns. Besides its uniqueness, the iris is a well protected organ and the iris patterns are stable over many decades; therefore the iris pattern is a suitable physical trait for the task of personal identification $[9,10]$.

\section{B. The Pupil}

The pupil is the dark opening around the center of the iris Generally, the center of the pupil is located slightly inferonasal to the center of the iris, measuring 2-4 $\mathrm{mm} \mathrm{[8].} \mathrm{Since} \mathrm{the} \mathrm{pupil}$ is slightly displaced toward the nasal side of the eye, the iris band on the temporal side (near the ear) is thus slightly wider than the iris band on the nasal side (near the nose).

An important function of the iris is the control of the size of the pupil, which is controlled by the dilator and sphincter pupillae muscles. In dim light, the dilator pupillae contracts, making the iris smaller and the pupil larger to allow more light into the eye. The dilation of the pupil occurs in dark conditions and when the body is in a state of excitement or fear. In bright light, the sphinter muscle fibers constrict the pupil, making the pupil smaller and the iris surface wider. The contraction of the pupil occurs in illuminated conditions, during convergence and while sleeping.

The reduction of the pupil size in light is known as the pupillary light reflex or pupillary reflex. The pupillary reflex is an involuntary and nearly instantaneous movement. The light reflex is also a consensual action, that is, when light illuminates one eye, the pupil of the other eye constricts along with the stimulated pupil. When the pupil becomes smaller, the iris spreads its tissues to compensate for the reduction of pupil size.

The constriction and dilation of the pupil may vary its size from 1-8 $\mathrm{mm}$ in diameter and there may be a slight degree of asymmetry between the right and left eye in normal individuals [8]. Loewenfeld and Newsome [11] reported a nonlinear arrangement of iris tissue occurs at the extreme of pupil response to strong light stimuli; the iris area between the collarette and pupil remains relatively constant as the pupil becomes smaller despite the increasing area of the iris. 


\section{IRIS DEFORMATION}

Iris deformation has been actively studied in the field of physiology for a long time. However, there is no general agreement about the mechanism. Some works $[12,13]$ reported that the iris surface is distorted by extension and compression parallel to the iris surface. Some surface deformation is handled by iris folds such as the circular contraction fold (or contraction furrow). Alternatively, Rohen [14] observed the iris collagen structure and suggested that the collagen fibers are arranged in a series of parallel arcs, joining the iris root and the pupil margin in a clockwise and counterclockwise angle of 90 degrees oriented by the center of the pupil, as shown in Fig. 5.

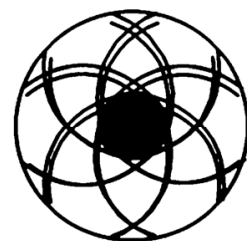

Fig.5. Meshwork skeleton of the iris [14].

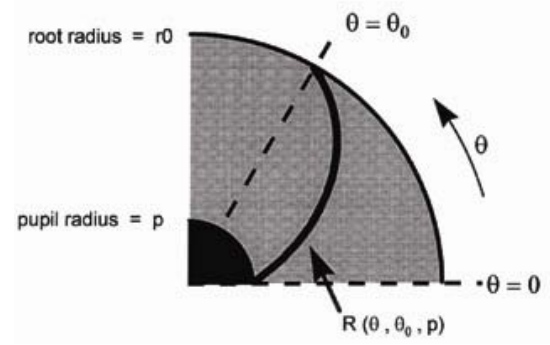

Fig.6. Nomenclature for describing an arc and irisrelated parameters in the iris [15].
Based on the structure of the iris suggested by Rohen and biological observations across a set of pupil sizes, Wyatt [15] proposed a nonlinear model called a minimum-wear-and-tear meshwork for the iris. The representation of the iris stretch is a combination of linear stretch $\left(R_{\text {linear }}\right)$ with additive non-linear stretch $(\Delta R(p, r))$. The representation is defined by $(1)$ :

$$
R_{\text {non-linear }}=R_{\text {linear }}+\Delta R(p, r)
$$

where $R_{\text {linear }}$ approximates the iris linear stretch under uniform lighting. It is a radius value defined by (2).

$$
R_{\text {linear }} \equiv R\left(\theta, \theta_{0}, p\right)=p_{\text {ref }}\left(\frac{R_{0}}{p_{\text {ref }}}\right)^{\theta / \theta_{0}} \frac{r_{0}-p}{r_{0}-p_{\text {ref }}}+\frac{p-p_{\text {ref }}}{r_{0}-p_{\text {ref }}},
$$

where $\theta$ is a polar angle coordinate depicted in Fig. $6 ; \theta_{0}$ is the polar angle traversed by a single fiber from the iris root to the pupil margin; $p$ is the radius of pupil, $p_{r e f}$ is a reference pupil radius and $r_{0}$ is the root radius. The additive non-linear function, $\Delta R($.$) , is a displacement of points related to its linear$ position. Wyatt suggested using the 6th order polynomial function defined by (3).

$$
\Delta R(p, r)=\Delta R(p, f)=\left(p-p_{r e f}\right)\left(r_{0}-p\right) \sum_{j=1}^{6} \frac{b_{j}}{j+1}\left(1-f^{j+1}\right),(3)
$$

where $f$ is a fractional position defined as:

$$
f=\frac{r_{0}-r}{r_{0}-p} .
$$

The value of $f$ is equal to zero at the iris $\operatorname{root}\left(r=r_{0}\right)$ and $f=1$ at the pupil margin. The coefficients $b_{j}$ were chosen so that $\Delta R=0$ at the iris root.

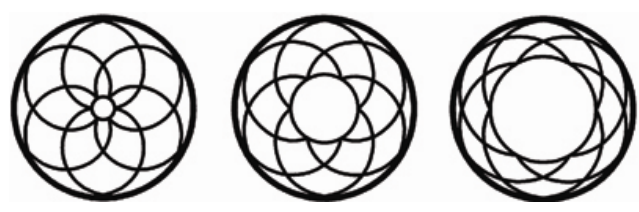

Fig.7. The optimized arcs of irises for $\theta_{0}=110^{\circ}$ and pupil diameters $=1.5,4.0$ and $7.0 \mathrm{~mm}[15]$.

An optimum form of the meshwork is then obtained by minimizing slips between the two-handed fiber directions, counterclockwise and clockwise, of the iris. The proposed optimization allows points along each fiber to move only in the radial direction as the pupil diameter varies. The experimental results indicated that the optimum mesh is reached when the polar angle traversed by a single fiber in going from the iris root to the margin $\left(\theta_{0}\right)$ is in the range of 100-110 degrees. Fig. 7 illustrated the optimized skeleton of the irises with various pupil sizes. Wyatt noted that the proposed meshwork model describes mainly the meshwork located in the posterior stroma of an iris. However, the anterior iris folds undergo remarkable stretching and compression much more than the posterior one.

Phang [16] studied iris surface deformation due to pupil movements by tracking the iris surface using Elastic Bunch Graph (EBG) matching. The tracking is performed over iris image sequences acquired in a dark room with adjustable illumination light sources. Since the iris has a circular-like shape and it mainly moves in radial directions, an elastic graph is placed such that its nodes are at the intersection points between iris radial lines and piecewise linear concentric circles, as shown in Fig. 8.

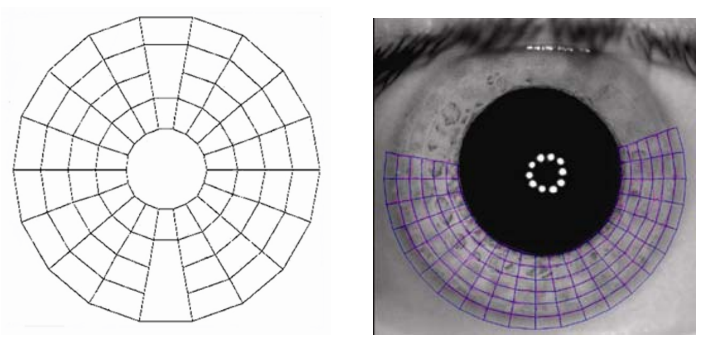

Fig.8. Diagrams of elastic graph nodes. [16]

Iris texture at each graph node is analyzed using Gabor filters. The iris texture is then tracked by comparing the similarity of Gabor's magnitude and phase across the iris image sequence. The tracking results indicate that movements of the iris surface occur mainly in the radial direction and the iris extends its surface area from the middle part of the iris during pupillary constriction. The cyclo-rotation around the visual axis of the eye is small, except at nodes located near the collarette and crypts. Thus, the stretch of the iris surface is nonlinear. However, the movement in radial locations of the iris surface features is approximately linear with the change of pupil size. Additionally, the iris surface deformation of each individual is not the same and there is a noticeable difference in iris deformation between the nasal side and the temporal side. Phang recommended confirming the experimental results with a larger database. 
Pamplona [17] analyzed iris deformation using a set of iris images captured under controlled lighting conditions. For each image, a series of iris feature points were manually marked and tracked along the set of acquired iris images. Fig. 9 shows an example of the tracked trajectories of the selected iris features. The obtained results confirm that the iris deformation occurs mainly in the radial direction. Most deviations from the radial path result from the existence of blood vessels under the iris and from crypts and folds. However, the amount of deviation is not substantial.

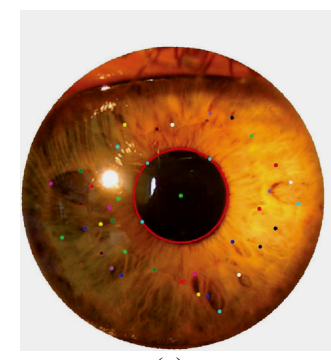

(a)

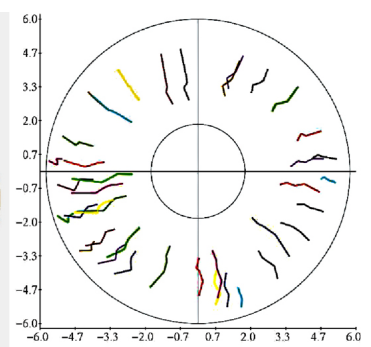

(b)
Fig.9. (a) An example of manually marked iris features. (b) Trajectory of the iris features across iris images sequence. [17]

\section{IRIS NORMALIZATION}

Usually, the acquired iris images of an iris recognition system contain irises of different sizes. Therefore, extracted features of one iris across the captured images are often positioned at different spatial locations. The unaligned features results in lower feature matching accuracy because of an iris recognition system identifies or verifies the identity of a person based on iris surface structure. The current-state-of-the-art iris recognition system generally compensates the deformation in a process called iris normalization. The process remaps the segmented iris ring to a fixed size rectangle. The linear mapping is often used in existing iris recognition systems.

\section{A. Daugman's Rubber Sheet Model}

The most common normalization method in the literature was proposed by Daugman [9, 10]. The Daugman's rubbersheet model transforms a segmented iris region, represented in the Cartesian coordinates system, into a fixed length and dimensionless polar coordinate system using the pupil center as a reference point. The method maps each point $(x, y)$ in the Cartesian domain to a point $(r, \theta)$ in polar coordinates, as shown in Fig. 10.
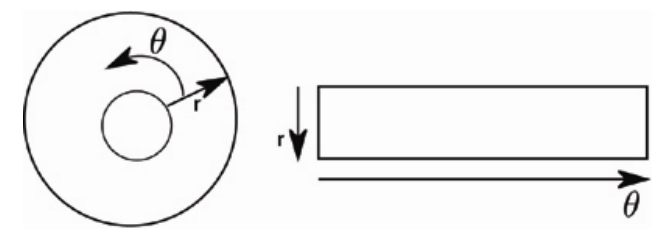

Fig.10. Rubber sheet model conversion.

The mapping equations, where $r$ represents the radial distance on the unit interval $[0 ; 1]$ and $\theta$ is an angle interval $[0,2 \pi]$, are

$$
\begin{aligned}
& x(r, \theta)=(1-r) x_{p}(\theta)+r x_{i}(\theta), \\
& y(r, \theta)=(1-r) y_{p}(\theta)+r y_{i}(\theta),
\end{aligned}
$$

where

$$
\begin{array}{ll}
x_{p}(\theta)=x_{p o}(\theta)+r_{p} \cos (\theta), & x_{i}(\theta)=x_{i o}(\theta)+r_{i} \cos (\theta), \\
y_{p}(\theta)=y_{p o}(\theta)+r_{p} \sin (\theta), & y_{i}(\theta)=y_{i o}(\theta)+r_{i} \sin (\theta),
\end{array}
$$

and $\left(x_{p o}, y_{p o}\right)$ are the coordinates of the pupil's center, $\left(x_{i o}, y_{i o}\right)$ are the coordinates of the iris's center, $\left(x_{p}, y_{p}\right)$ are the coordinates of the pupil boundary, and $\left(x_{i}, y_{i}\right)$ are the coordinates of the iris boundary along the $\theta$ direction.

The rubber-sheet model is a linear mapping function. It accounts for scale and translation variants. The rotation variant is addressed in the matching stage by selecting the best match among the $n$-shift iris template comparisons since rotation of an iris in the Cartesian coordinates is equivalent to a shift in the polar coordinates. Several normalization methods are similar to this. The only difference is the selection of reference points which can be the center of the pupil or the iris or a virtual center.

\section{B. Wilde's Image Registration}

Wilde's [18] compensated the iris deformation using an image registration technique. The technique searches for an optimal transformation in both space and intensity that maps each point from one image to a point in the other. In [18], a common affine transform that maps straight lines into straight lines denoted by (7) is used.

$$
\left(\begin{array}{l}
x^{\prime} \\
y^{\prime}
\end{array}\right)=\left(\begin{array}{l}
x \\
y
\end{array}\right)-s R(\theta)\left(\begin{array}{l}
x \\
y
\end{array}\right) .
$$

The transformation compensates for scaling $s$ and rotation by $\theta, R(\theta)$. The transformation maps points in a newly acquired image, $I_{a}(u, v)$ with points of an image in the database, $I_{d}(u, v)$ such that it would minimize an objective function defined by (8). The two parameters, $s$ and $R(\theta)$ are recovered via an iterative minimization procedure.

$$
\iint_{x}\left(\left(I_{d}(x, y)-I_{a}(x-u, y-v)\right)^{2} d x d y\right.
$$

Similar to Wilde's approach, Ivin [19] proposed using two scaling parameters, a global and a local one, for an iris deformation model. He described the state of the iris deformation using the five-parameter transformation matrix defined by (9).

$$
\left(\begin{array}{l}
x \\
y
\end{array}\right) \rightarrow \delta(r) R(\theta)\left(\begin{array}{l}
x-c_{x} \\
y-c_{y}
\end{array}\right)+\left(\begin{array}{l}
t_{x}-c_{x} \\
t_{y}-c_{y}
\end{array}\right),
$$

where $(x, y)$ are the coordinates in the initial iris image, $\left(c_{x}, c_{y}\right)$ is the centroid of the model, which is computed from the coordinates of the $M$ samples in the iris signatures, and $\delta(r)=1$ $+\delta_{1}+\delta_{2}$. The five parameters are rotation $R(\theta)$, translation $\left(t_{x}, t_{y}\right)$ in $x$ - and $y$ - coordinates and two scaling parameters: a uniform global scaling, $\delta_{l}$, and a radial scaling across iris, $\delta_{2}$, which represents pupil contraction and expansion. 


\section{Non-linear Iris Normalization}

Studies of iris physiology reported that the response of the texture with respect to different intensities of light is non-linear due to the distribution of iris muscles controlling the pupil size [20]. Therefore, later works of iris normalization incorporate non-linear iris normalization.

Yuan and Shi [21] proposed non-linear normalization methods based on the structure of iris fibers proposed by Wyatt. The iris arcs are modeled over a normalized pupil. All irises are first mapped such that a predefined $\lambda=r / R$ is maintained, where $r$ and $R$ denote the radius of the pupil and limbus, respectively. The predefined $\lambda$ is the mean value of all $\lambda$ values obtained from iris images in a database. The virtual iris arcs connect one point on the pupil margin to another point on the iris root. The polar angle traversing along these two points is $\pi / 2$, as depicted in Fig. 11 .

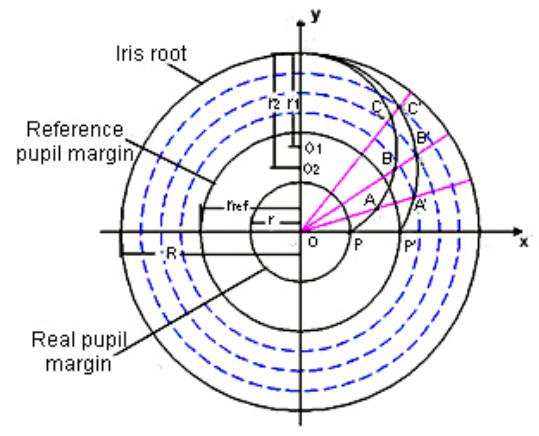

Fig.11. Diagram of iris skeletons.

When the pupil changes its size from $r_{r e f}$ to $r$, point $A^{\prime}\left(x_{A^{\prime}}\right.$, $\left.y_{A^{\prime}}\right)$ moves to point $A\left(x_{A}, y_{A}\right)$, which can be computed using the following equations:

$$
\begin{aligned}
& y-y_{A^{\prime}}=k\left(x-x_{A^{\prime}}\right) \\
& x^{2}+\left(y-y_{1}\right)^{2}=r_{1}^{2}
\end{aligned}
$$

where $k=y_{A^{\prime}} / x_{A^{\prime}}, y_{1}=\left(R^{2}-r^{2}\right) / 2 R$ and $r_{1}=\left(R^{2}+r^{2}\right) / 2 R$.

Wei et. al. [22] proposed an alternative non-linear iris normalization model based on statistical learning. The proposed iris deformation model is also a combination of linear and non-linear stretch described by (1), where the linear stretch is defined by (2). However, the non-linear stretch, $\Delta R$, is modeled using a Gaussian function with parameters obtained by training. The proposed method was validated using two databases: DB1 [22] and CASIAv.3-Lamp [23]. The DB1 database contains 30 iris classes. The CASIAv.3-Lamp iris database contains 819 classes, but only 400 classes were used in the experiment.

To evaluate the system performance, equal error rates (EER) and decidability index (DI) of the system are computed and compared with results obtained using iris normalization proposed by Daugman [9] and with the method in [21]. The EER is an equal error rate at which the false reject rate (FRR) is equal to the false accept rate (FAR). A false reject occurs when a system rejects a valid identity; a false accept occurs when a system falsely accepts an identity. The DI measures the separation among authentic and imposter distributions. The measure is defined by

$$
D I=\frac{\left|\mu_{A}-\mu_{I}\right|}{\sqrt{\left(\sigma_{A}^{2}+\sigma_{I}^{2}\right) / 2}},
$$

where $\mu_{A}$ and $\mu_{I}$ are the means of the authentic and imposter distributions, the $\sigma_{A}$ and $\sigma_{I}$ are the related standard deviations of the two distributions. The system comparisons are shown in Table 1. According to the results, the iris deformation correction via statistic learning improved the performance of iris recognition systems.

TABLE I. EER AND DI COMPARISONS [22]

\begin{tabular}{|c|c|c|c|c|}
\hline Database & Error & Daugman & Yuan & Wei \\
\hline \multirow{2}{*}{ DB1 } & EER & $1.058 \%$ & $0.857 \%$ & $0.733 \%$ \\
\cline { 2 - 5 } & $\boldsymbol{D I}$ & 4.7094 & 4.8213 & 4.9913 \\
\hline $\begin{array}{c}\text { CSIAv.3- } \\
\text { Lamp }\end{array}$ & $\boldsymbol{E E R}$ & $0.973 \%$ & $0.831 \%$ & $0.719 \%$ \\
\cline { 2 - 5 } & $\boldsymbol{D I}$ & 4.7509 & 4.8409 & 4.9083 \\
\hline
\end{tabular}

\section{DISCUSSION AND CONCLUSION}

To date, researchers in the field of iris recognition have attempted to address iris pattern distortion caused by changes in the size of the pupil due to illumination variations. Recently proposed iris normalization procedures accommodate size variations by including non-linear terms within the model. However, there is still a need to further investigate iris surface deformation under changes in lighting conditions in order to obtain more accurate and reliable iris normalization procedures.

The histology of the iris also suggests that deformation over the whole iris surface is non-uniform. This is due to the constriction of the two muscles of the iris (sphincter and dilator muscle) affecting the surface deformation differently. Thus, a separate study of iris surface deformation over pupillary and ciliary zones may improve understanding of the phenomenon. However, tracking iris features in the pupillary zone is very challenging since iris features are highly redundant within this zone. The other major challenge in iris deformation study is to establish large common iris image databases that allow the study of iris surface deformation and of feature tracking and recognition in general to compare and contrast to each other.

Due to iris textures appears differently under different lighting sources, establishing multi-spectral iris image database allows fusing complementary information to improve feature tracking performance. Examples are iris texture of dark color irises is more visible under NIR light than under visible light whereas fine iris texture of light color irises appears clearer in color iris images captured under visible light source. The color iris images also provides better depth information of iris texture. Furthermore, in-depth investigation of surface deformation can be done with iris images acquired using slitlamp microscopy since this device allows users to adjust the light beam and position, revealing clearer small iris structures.

In conclusion, this paper presents the interdisciplinary background relevant to research on iris surface deformation and iris normalization with an emphasis on physiologically 
based approaches. An in-depth investigation on iris surface deformation will result in a more accurate and reliable iris recognition system.

\section{ACKNOWLEDGMENT}

We would like to thank Assoc. Prof. Jutalai Tanterdtham from Siriraj Hospital, Mahidol University, for proofreading section II of this paper. Portions of the research in this paper use the CASIA-IrisV4 collected by the Chinese Academy of Sciences' Institute of Automation (CASIA).

\section{REFERENCES}

[1] K. Hollingsworth, K. W. Bowyer, P. J. Flynn, "Pupil dilation degrades iris biometric performance," Computer Vision and Image Understanding, vol. 113, 2009, pp. 150-157.

[2] L. Ma, T. Tan, Y. Wang, D. Zhang, "Efficient iris recognition by characterizing key local variations," IEEE Trans. on Image Processing, vol. 13 , no. 6,2004 , pp. 739-750.

[3] A. Muron, J. Pospisil, "The human iris structure and its usages," Acta Univ. Palacki Phisica, vol. 39, 2000, pp. 87-95.

[4] P. J. Caroline, M. P. André, "The effect of corneal diameter on soft lens fitting, part 1," Contact Lens Spectrum, vol. 17, no. 4, 2002, pp. 56.

[5] J. V. Forrester, A. D. Dick, P. G. McMenamin, W. R. Lee, "The eye: Basic sciences in practice," W.B. Saunders, 2001.

[6] N. Davis-Silberman, R. Ashery-Padan, "Iris development vertebrates; genetic and molecular considerations," Brain Research, vol. 1192, 20085, pp. 17-28.

[7] R. C. Eagle Jr, "Iris pigmentation and pigmented lesions: An ultrastructural study," Trans. of the American Ophthalmological Society, vol. 86, 1988, pp. 581-687.

[8] D. H. Cherwek, H. E. Grossniklaus, A. K. Hutchinson, "Iris,Duane's foundations of clinical ophthalmology: Volume 1," Lippincott Williams \& Wilkins, 2006.

[9] J. Daugman, "High confidence visual recognition of persons by a test of statistical independence," IEEE Trans. on Pattern Analysis and Machine Intelligence, vol. 15, 1993, pp. 1148-1161.
[10] J. Daugman, "How iris recognition works," International Conference on Image Processing, vol. 1, 2002, pp. 33-36.

[11] I. Loewenfeld, D. Newsome, "Iris Mechanics: I. Influence of Pupil Size on Dynamics of Pupillary Movements," American Journal of Ophthalmology, vol. 71, 1971, pp. 347-362.

[12] M. Hogan, J. Alvarado, J. Weddell, "Histology of the human eye," Saunders, Philadelphia, 1971.

[13] O. Lowenstedn and I. Loewenfeld, "The pupil, muscular mechanisms," Davson, H. (Ed.), 2nd edition., vol. 3, Academic Press, pp. 255- 337, 1969.

[14] H. Rohen, "Der bau der regenbogenhaut beim menschen und einigen saugern," Gegenbaur Morphology Journal, vol. 91, 1951, pp. 140-181.

[15] H. J. Wyatt, "A 'Minimum-Wear-and-Tear' meshwork for the iris," Vision Research, vol. 40, no. 16, 2000, pp. 2167-2176.

[16] S. S. Phang, "Investigating and developing a model for iris changes under varied lighting conditions," Master thesis, School of Engineering Systems, Faculty of Built Environment and Engineering, Queensland University of Technology, 2007.

[17] V. F. Pamplona, M. M. Oliveira, G. V. G. Baranoski, "Photorealistic models for pupil light reflex and iridal pattern deformation," ACM Trans. Graph, vol. 28, no. 4, 2009, pp. 106:1-106:12.

[18] R. Wildes, "Iris Recognition: An emerging biometric technology," Proceedings of the IEEE, vol. 85, no. 9, 1997, pp. 1348-1363.

[19] J. P. Ivins, J. Porrill, "A deformable model of the human iris for measuring small three-dimensional eye movements," Machine Vision and Applications, vol. 11, no. 1, 1998, pp. 42-51.

[20] P. Bressloff, C. Wood, P. Howarth, "Nonlinear shunting model of the pupil light reflex," Proceedings of the Royal Society B: Biological Sciences, vol.263, no.1373, 1996, pp. 953-960.

[21] X. Yuan, P. Shi, "A non-linear normalization model for iris recognition," Advances in Biometric Person Authentication, Springer, 2005, pp. 135-141.

[22] Z. Wei, T. Tan, Z. Sun, "Nonlinear iris deformation correction based on Gaussian model," Advances in Biometrics, Springer, 2007, pp. 780-789.

[23] CASIA iris image database, 2012, avilable at http://biometrics.idealtest.org. 